\title{
Comparison of health measures to movement data in aware homes
}

\author{
Brian O’Mullane, Brennon Bortz, Ann O’Hannlon, John Loane, R. Benjamin \\ Knapp \\ CASALA / Netwell, PJ Carrolls Building, DkIT, Ireland \\ \{brian.omullane, brennon.bortz, john.loane, ben.knapp\}@casala.ie, \\ ann.ohannlon@netwellcentre.org
}

\begin{abstract}
Detecting wellness in older adults with just ambient sensors is a challenging and difficult task, one that can only be address with large volumes of detailed annotated data and a diverse participant base. Presented here are early results comparing movement data to baseline depression and mobility data from a purpose built 16 unit ambient assisted living development in Ireland. With the goal of ultimately detecting health changes in an older population with ambient sensors, results here show that whereas there is some correlations between health measures and sensor data as well as some observable patterns, but more work needs to be done.
\end{abstract}

Keywords: AAL, Aware Homes, KNX, Depression, Mobility, PIR

\section{Introduction}

The population is living longer and with this there is a push towards improving quality of life of older people as well as allowing them control and autonomy while aging. Nine years ago, female life expectancy in the record-holding country (Japan), had risen for 160 years at a steady pace of almost three months per year, and this upward trend has continued[1]. Ambient assisted living offers a potential solution to this problem and hence is an active area of research. It involves embedding low impact pervasive sensors, such as presence sensors and door usage sensors in homes. Using this technology to help build a picture of behaviour and detecting when this behaviour changes over time, and correlating this to indicators of decline is the ultimate goal of this research. But what does this behaviour presented by the sensors look like? How can we relate this behaviour to physical and mental health issues? In this paper we present data gathered from ambient sensors embedded the homes of 10 older people over a 3-month period. We look at the movement behaviour of the residents as well as baseline measures of their depression and mobility and try to identify commonalities between them.

\section{Background and related work}

A primary activity at the Centre for Affective Solutions for Ambient Living Awareness (CASALA) is our work with a number of older adults living at the Great 
Northern Haven (GNH) demonstration housing project. GNH consists of 16 purposebuilt aware homes in Dundalk, Ireland. Each home is equipped with a combination of sensor and interactive technologies to support ambient assisted living for older people. Currently there are 13 homes occupied by 11 men and 4 women. Using a total of 2240 sensors, actuators and higher level alerts throughout the development, we have collected close to 100 million records of data to date. The sensors include presence sensors, contact sensors on all internal and external doors and windows, electricity, water and heating usage sensors, ambient light and temperature sensors as well as an array of other ambient sensors. For this study we are examining movement behaviour, using just the presence sensors in the hallway, bedroom, living room and the door sensor for the main entrance. The presence sensors are KNX passive infrared (PIR) sensors tuned to give readings of any small movement in a room with a reset interval of ten seconds.

This paper focuses on a first look at features that could be used as part of a behaviour recognition engine, currently in development[2], and compares them to baseline health measures taken from residents.

\section{Data Description}

The cohort for this study begun moving in to their homes in June 2010. Two of the three PIRs used for this study were moved from their location over the window to a new location over the entry door in the bedroom and livingroom on April $13^{\text {th }}$, to ensure capture of entry in to those rooms. Hence for this paper, only readings between $19^{\text {th }}$ of April and $22^{\text {nd }}$ of July 2011 are considered. All of the data used in this study was gathered KNX sensors and aggregated and logged by NETxAutomation OPC server software. Two of the homes are occupied by two residents - data for these homes were removed from the examination as the presence for each resident is difficult to determine and outside the scope of this study. Difficulties with incomplete data was observed on 3 of the 92 days, by examining a periodic sensor data (power sensor samples at $0.1 \mathrm{~Hz}$ ), these 3 days were moved entirely from the dataset.

The layout of the homes are show in figure 1 . The resident must pass through the hall way sensor when moving between any of the rooms, such as when they are visiting the main bathroom from the living area during the day.

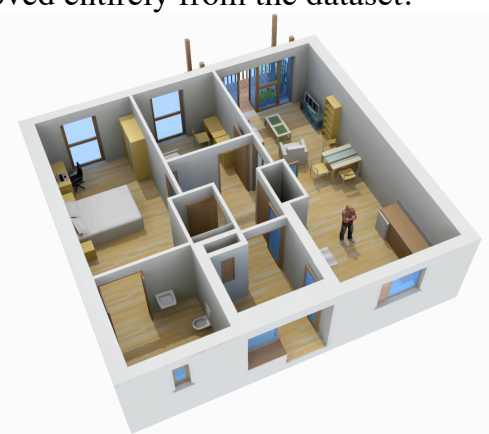

Figure 1 - Great Northern Haven home layout

Each presence sensor fired on average 155 times a day and the sensor firing times gave a characteristic that is easily identifiable by manual inspection. To help visualise behaviour patterns the sensor data was represented on a spiral plot called a "last 
clock"[3] that plots the data on a 24-hour clock with midnight at the top and spirals out from the centre. Each circuit represents a day.

The outing data was derived from both the front door sensor as well as the PIR inside home data. It was simply deduced from door firing and no movement inside house followed by another door firing. Outings group in to two very distinct groups which can be easily separated, outings of less than 5minutes are considered to be less significant and removed from this study. The design of the apartments is such that all of the residents on the ground flour have a second outside door operated by swipe card, the door sensor is located on the inside or apartment door, but inspecting the data shows unusually high number of firings for the apartment doors for 2 (ID4,5) of the 3 residents in the ground floor apartments. A characteristic of the KNX door sensors used is that they fire periodically if a door is left open, this high number of firings might suggest this internal door was left open. Additionally the hall PIR for apartment ID 25 reported no firings during this time, hence could be concluded as either damaged or incorrectly addressed.

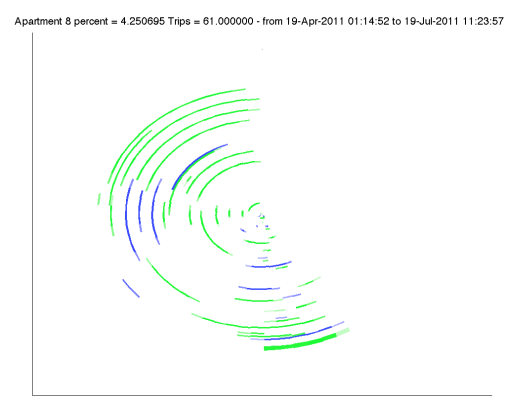

figure 2 Clock plot of time outside home for person with lowest depression score

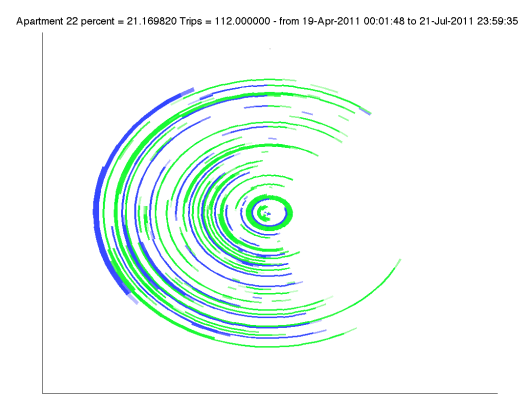

figure 3 Clock plot of time outside home for person with highest depression score

Holiday data was derived from PIR data falling below a very low number for a resident in a day, the number chosen was 20 , where normal daily average was 465 . Holiday data was used to remove holidays from time outside and PIR firing analysis.

Mobility status: Mobility was assessed using the walking subscale of the Health Assessment Questionnaire Disability Index (HAQ-DI)[4]. The HAQ-DI assesses physical functioning in upper and / or lower extremities. The walking subscale, participants were asked about their ability to walk outdoors on flat ground and to climb up five steps. Response options ranged from zero (self-sufficient) to three (severely disabled). 
Table 1. Mobility data. Understandably time outside show's strong correlation with mobility measure. $\mathrm{P}=.057$

\begin{tabular}{cccccc}
\hline Apt ID & Mobility & Total PIR Records & Holidays & Time Outside (\%) & Outings \\
\hline 4 & 1 & 25505 & 0 & 19.64 & $1775^{1}$ \\
5 & 1 & 32649 & 4 & 16.67 & $1092^{1}$ \\
20 & 1 & 47659 & 0 & 10.18 & 238 \\
22 & 1 & 48222 & 2 & 21.16 & 112 \\
25 & 1 & 28299 & 0 & 30.5 & 247 \\
7 & 2 & 25381 & 0 & 1.79 & 59 \\
11 & 2 & 17696 & 7 & 10.17 & $155^{1}$ \\
8 & 2.7 & 41570 & 2 & 4.25 & 61 \\
3 & No Data & 4048 & 52 & 4.29 & $112^{1}$ \\
12 & No Data & 43012 & 0 & 3.04 & 83 \\
\hline
\end{tabular}

Psychopathology: To examine mental health or well-being the General Health Questionnaire (GHQ-12) was used[5]. This measure consists of 12 items relating to common mental health experiences of depression, anxiety, somatic symptoms and social withdrawal. It is a screen to identify respondents likely to have or be at risk of developing psychiatric disorders. This scale is used widely in studies of well-being [6]. In the current study, higher scores equate with higher levels of psychopathology.

With the goal of comparing sleep patterns to depression scores bedroom PIRs sensor data was examined. It was hoped the standard deviation of bedroom presence data could help determine sleep times, but the data proved too inconsistent.

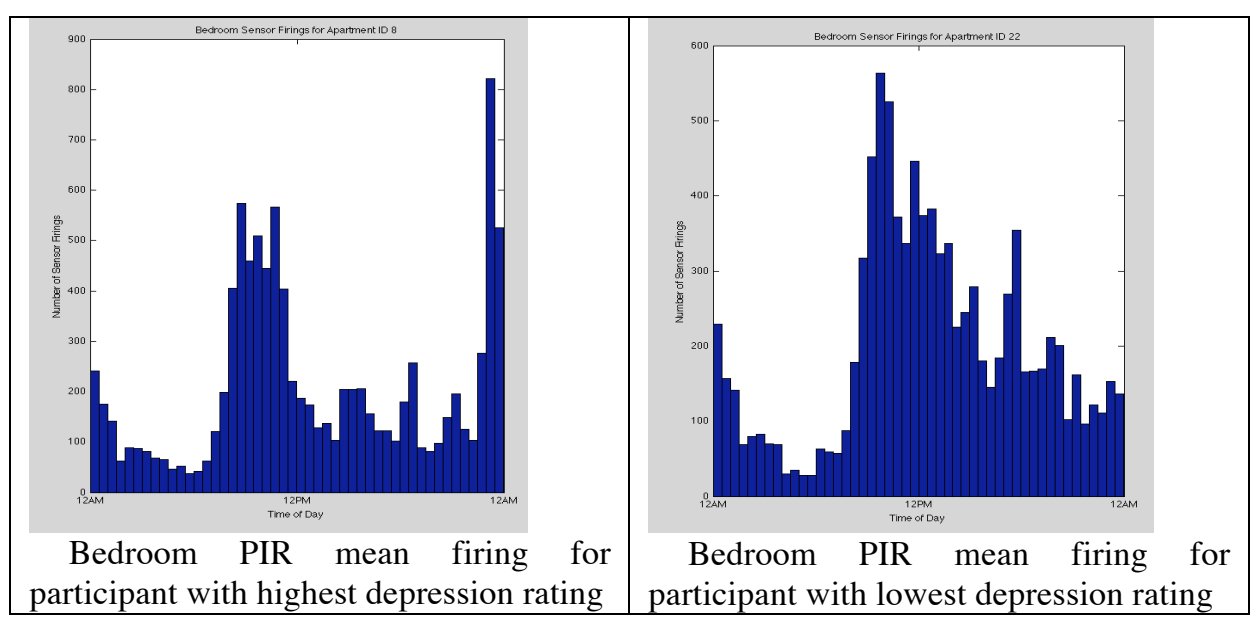

${ }^{1}$ Ground floor apartment with extra security door allowing apartment door to be left open, outing data cannot be trusted 
Table 2. Depression Data. Only weak correlations to presence patterns observed.

\begin{tabular}{|c|c|c|c|c|c|c|c|}
\hline & & \multicolumn{6}{|c|}{ Mean Daily PIR firing } \\
\hline $\begin{array}{l}\text { Apt } \\
\text { ID }\end{array}$ & Depression & $\begin{array}{l}\text { Time } \\
\text { outside } \\
(\%)\end{array}$ & Outings & Bedroom & Hall & $\begin{array}{l}\text { Living } \\
\text { Room }\end{array}$ & $\begin{array}{l}\text { STD of } \\
\text { time in } \\
\text { bedroom }\end{array}$ \\
\hline 8 & 19 & 4.25 & 61 & 113.82 & 71.23 & 292.77 & 0.27 \\
\hline 5 & 22 & $16.67^{1}$ & $1092^{1}$ & 25.98 & 79.52 & 278.60 & 0.22 \\
\hline 11 & 23 & 10.17 & 155 & 65.46 & 30.62 & 119.72 & 0.21 \\
\hline 25 & 24 & 30.50 & 247 & 91.89 & $0.00^{2}$ & 226.08 & 0.20 \\
\hline 3 & 25 & $4.29^{1}$ & $112^{1}$ & 319.65 & 236.89 & 535.57 & 0.27 \\
\hline 22 & 30 & 21.16 & 112 & 110.01 & 120.11 & 324.15 & 0.23 \\
\hline
\end{tabular}

\section{Discussion and Further Work}

It is dangerous to draw any firm conclusions on a test group with $\mathrm{n}=10$, particularly where data is complete for only 3 residents, but with sensor data in this study of over 300,000 records some interesting results can be anticipated. Bedroom sensors show observable peaks in at bedtime and morning time, with further analysis sleeping hours may be determined. Outings and time out of home cannot be ruled out of correlations with depression, in fact the data tends towards the opposite. Mobility questionnaire correlates very well with what is observed by the sensors.

More baseline depression data will strengthen result conclusions, and better validation and monitoring of KNX sensors is required.

Acknowledgments. To Florian Le Touzé and Diane Fauré, research assistants at CASALA, for data analysis and Enterprise Ireland's Applied Research Enhancement Programme with support from EU Structural funds.

\section{References}

[1] J. Oeppen and J. W. Vaupel, "Broken limits to life expectancy," Science, vol. 296, no. 5570, p. 1029, 2002.

[2] J. Loane, B. O’Mullane, B. Bortz, and R. B. Knapp, "Interpreting Presence Sensor Data and Looking for Similarities Between Homes Using Cluster Analysis," presented at the Pervasive health 2011.

[3] J. Angesleva and R. Cooper, "Last clock," IEEE Computer Graphics and Applications, vol. 25 , no. 1 , pp. 20-23, 2005.

[4] J. Fries, P. Spitz, and D. Young, "The dimensions of health outcomes: the health assessment questionnaire, disability and pain scales.," J Rheumatol, vol. 9, no. 5, pp. 789-93, 1982.

[5] D. P. Goldberg, Manual of the General Health Questionnaire. NFER Publishing.

[6] M. Jones, "The burden of psychological symptoms in UK Armed Forces," Occupational Medicine, vol. 56, no. 5, pp. 322-328, 2006.

${ }^{2}$ Damaged PIR 